Gestión y Estrategia

Management and Strategy

Gestão e Estratégia

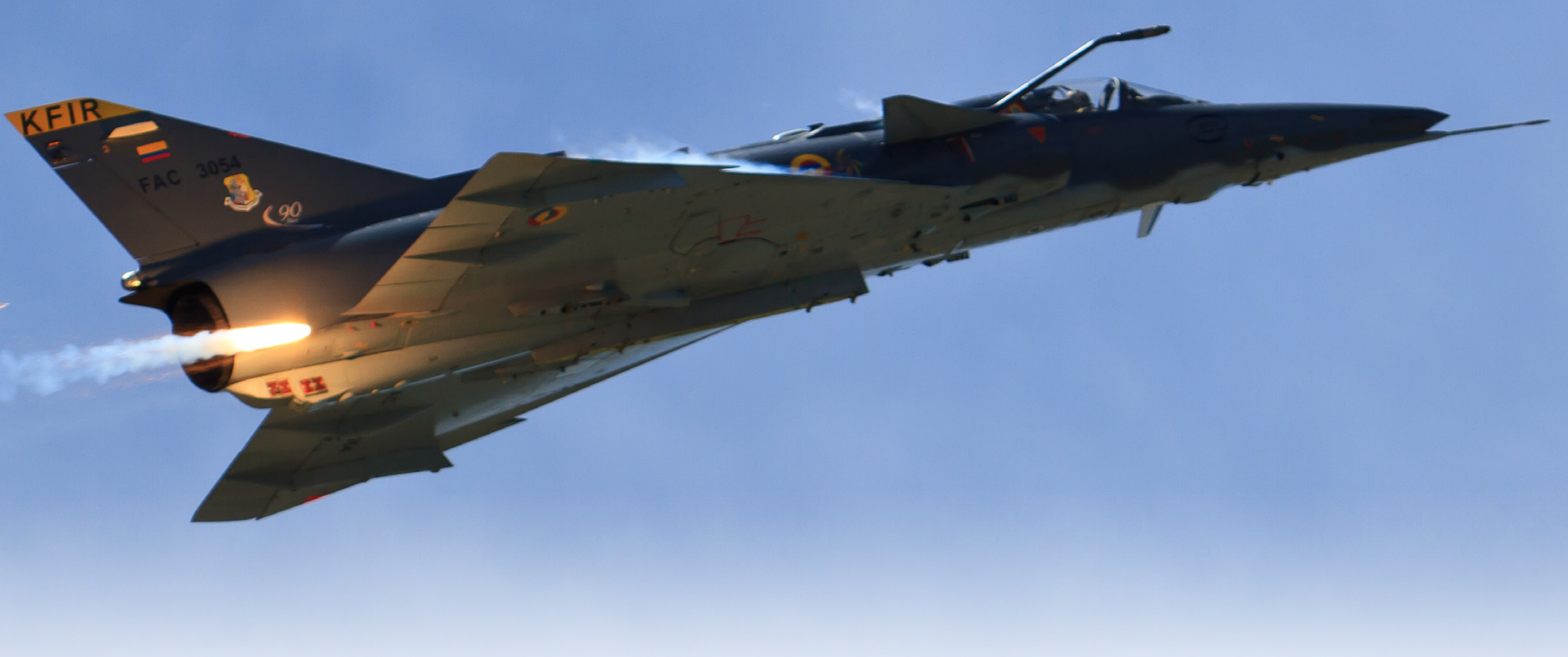

La Seguridad Multidimensional y el Poder Aéreo: doctrinas de la Oea y Fuerza Aérea para fortalecer el desarrollo de la Seguridad y La Defensa. ¿Cuál es el nuevo panorama de Colombia?'

Multi-dimensional security and Aerial Power: OAS and Air Force Doctrines to Strength the Development of Security and Defense: What is Colombia's New Panorama? ${ }^{3}$

Segurança Multidimensional e o Poder Aéreo: doutrinas da OEA e da Força Aérea para fortalecer

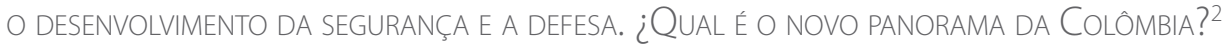

David Barrero Barrero ${ }^{4}$,Fabio Baquero Valdés ${ }^{5}$,Andrés Gaitán Rodríguez ${ }^{6}$ Escuela Superior de Guerra. Bogotá, Colombia

Universidad Santo Tomás. Bogotá, Colombia

\title{
CIENCIA Y PODER AÉREO
}

ISSN 1909-7050 / E- ISSN 2389-2468 / Volumen 13 / Número 1 / Enero-Junio de 2018 / Colombia / pp. $72-81$ Recibido: 11/09/2017

Aprobado par evaluador: 08/04/2018

Doi: https://doi.org/10.18667/cienciaypoderaereo.587

72 | CIENCIA Y PODER AÉREO | Revista Científica de la Escuela de Postgrados de la Fuerza Aérea Colombiana 
Para citar este artículo:

Insulza, J. M., Barrero, D., Baquero Valdés, F. y Gaitán Rodríguez, A. (2018). La Seguridad Multidimensional y el Poder Aéreo: doctrinas de la OEA y Fuerza Aérea para fortalecer el desarrollo de la seguridad y la defensa. ¿Cuál es el nuevo panorama de Colombia?. Ciencia y Poder Aéreo, 13(1), 72-81. doi: https://doi.org/10.18667/cienciaypoderaereo.587.

' Este artículo es producto del proyecto de investigación denominado "La Seguridad Multidimensional y el Poder Aéreo: doctrinas de la OEA y Fuerza Aérea para fortalecer el desarrollo de la seguridad y la defensa. ¿Cuál es el nuevo panorama de Colombia?". A cargo del Departamento de Fuerza Aérea y financiado por la Escuela Superior de Guerra, adscrito al grupo de investigación Masa Crítica.

${ }^{2}$ Original article derived from the research project named "Multi-dimensional Security and Aerial Power: OAS and Air Force Doctrines to Strength the Development of Security and Defense: What is Colombia's New Panorama?", led by Air Force Department and funded by Escuela Superior de Guerra, attached to the research group Masa Crítica.

${ }^{3}$ Artigo original produto do projeto de pesquisa denominado "A Segurança Multidimensional e Poder Aéreo: doutrinas da OEA e Força Aérea para fortalecer o desenvolvimento da segurança e da defesa. ¿Qual é o novo panorama da Colômbia?" Responsável pelo Departamento da Força Aérea e financiado pela Escola Superior de Guerra, ligado ao grupo de pesquisa Masa Crítica.

${ }^{4}$ Magíster en Ciencias de la Seguridad y Defensa Interamericana del Colegio Interamericano de Defensa. Docente e investigador de la Escuela Superior de Guerra en las cátedras de Fundamentos de Lógica Estratégica al Curso de Altos Estudios Militares y Campos de Acción del Estado y Doctrina y Operaciones Aéreas a los Cursos de Estado Mayor. Docente en la especialización de Administración Aeronáutica de la Universidad Militar Nueva Granada. Coronel de la Reserva Activa de la Fuerza Aérea Colombiana, piloto y administrador aeronáutico. Correo electrónico: davidbarrerob@gmail.com

${ }^{5}$ Administrador Aeronáutico, Especialista en Seguridad y Defensa Nacional, Gerencia Estratégica de Costos y Empresarial y Control Interno. Auditor Líder de las Normas ISO y NTSGP-1000. Especialista en docencia universitaria, par académico del CNA y Magíster en Educación de la Universidad Santo Tomás. Coronel de la Reserva Activa de la Fuerza Aérea Colombiana. Correo electrónico: crlfabiob@gmail.com

${ }^{6}$ Politólogo e internacionalista de la Pontificia Universidad Javeriana. Magíster en Defensa y Seguridad Nacional. Docente de planta de la Universidad Santo Tomás. Investigador del Departamento de la Escuela Superior de Guerra. Correo electrónico: andresgaitan@usantotomas.edu.co
Resumen: El presente artículo de investigación aborda la temática de la seguridad y defensa de las naciones desde la perspectiva de empleo del Poder Aéreo en Colombia frente a los retos de la Seguridad Multidimensional. Para este propósito se aplicó la metodología de revisión y análisis documental, la que permitió efectuar un examen comparativo de los elementos teóricos, conceptuales y prácticos del Poder Aéreo a partir de tres ejes temáticos específicos. El primero relacionado con las doctrinas de Poder Aéreo en Colombia y las amenazas tradicionales a la Seguridad Multidimensional, el segundo en función a la necesidad de articular el empleo del Poder Aéreo a las nuevas amenazas y sus características a partir de la globalización y aplicación de las TIC, el tercero relacionado con la armonía de empleo del Poder Aéreo frente a los escenarios de preocupaciones y nuevos desafíos a la seguridad, elemento donde reside la postura más revolucionaria del concepto de Seguridad Multidimensional. El anterior proceso facilitó la deducción de hallazgos y la deducción de importantes conclusiones dando respuesta al planteamiento problémico.

Palabras clave: Seguridad Multidimensional, Poder Aéreo, Fuerza Aérea Colombiana, doctrina, nuevas amenazas, amenazas tradicionales, preocupaciones y otros desafíos

Abstract: This research article approaches the issue of security and the defense of the nations from the perspective of the Aerial Power use in Colombia before the challenges of multi-dimensional security. For this purpose, the methodology of revision and documentary analysis was applied, which allowed to make a comparative exam of the theoretical, conceptual, and practical elements of Aerial Power from three specific topics. The first of them is that related to the doctrine of Aerial Power in Colombia and the traditional threats to multi-dimensional security; the second relies in the need to articulate the use of aerial power in contexts of preoccupations and new challenges to security, in which relies the most revolutionary position of the concept of multidimensional security. The former process facilitated findings deduction and drawing important conclusions, giving answers to the problem that was stated.

Keywords: Multi-dimensional Security, Aerial Power, Colombian Air Force, Doctrine, New Threats, Traditional Threats, Preoccupations, New Challenges

Resumo: O presente artigo de pesquisa aborda a questão da segurança e defesa das nações a partir da perspectiva do uso do Poder Aéreo na Colômbia diante dos desafios da Segurança Multidimensional. Para este objetivo, aplicou-se a metodologia de revisão e análise documental, a que permitiu fazer um exame comparativo dos elementos teóricos, conceituais e práticos do Poder Aéreo a partir de três eixos temáticos específicos. A primeira relacionada com as doutrinas do Poder Aéreo na Colômbia e as ameaças tradicionais à Segurança Multidimensional, o segundo de acordo com a necessidade de coordenar o uso do Poder Aéreo às novas ameaças e suas características a partir da globalização e aplicação das TIC, a terceira relacionada à harmonia do uso do Poder Aéreo diante dos cenários de preocupações e novos desafios à segurança, elemento onde reside a postura mais revolucionária do conceito de Segurança Multidimensional. O processo anterior facilitou a dedução dos achados e a dedução de conclusões importantes em resposta à abordagem problemática.

Palavras-chave: Segurança Multidimensional, Poder Aéreo, Força Aérea Colombiana, doutrina, novas ameaças, ameaças tradicionais, preocupações e outros desafios. 
"No tenemos guerras en la región, pero sí dos enemigos comunes e interrelacionados, y a cual más violento: el crimen organizado y la desigualdad".

José Miguel Insulza Secretario General de la Organización de Estados Americanos por dos periodos consecutivos, del 2005 al 2015.

\section{Introducción}

La seguridad' como concepto y práctica (deber) de los Estados-nación ha mutado a lo largo de la historia; incluso, si solo se observa desde el periodo de la modernidad hasta la actualidad. En una primera "fase", el concepto de amenaza como el de seguridad se ha definido desde un enfoque unidimensional emanado de la amenaza o realización de la "guerra interestatal".

A partir de la Guerra Fría surgen actores armados subversivos y criminales (que detentaron el monopolio constitucional de la violencia al Estado). Ahora bien, con la llegada de la década de los 90 (siglo XX) y junto con la globalización y las Tecnologías de la Información y la Comunicación (en adelante: TIC) se transformaron de formas locales a formas de organización trasnacional, caracterizándose dentro del conjunto de las "nuevas amenazas".

De esta manera, la seguridad se ha convertido en un elemento más difuso de determinar, y así mismo, más difícil de garantizar como valor. No obstante, una tendencia propia del siglo XXI es una categoría de análisis denominada como "las preocupaciones y otros desafíos" a la seguridad; espacio en el cual ya ni siquiera se plantean problemáticas derivadas del uso de la violencia (legal e ilegal) o las armas, sino de fenómenos como los desastres naturales, las pandemias, el deterioro del medio ambiente, etc. Así, y en el caso de Colombia, siendo una institución que se ha formado y evolucionado en mayor medida para responder a un escenario de inseguridad interno (conflicto armado colombiano).

Razones por las cuales el objetivo de este artículo es, en primera medida, exponer el proceso evolutivo que ha presentado la seguridad en la modernidad; esto, como producto no solo de los cambios políticos en las relaciones de los Estados, sino por la aparición de nuevos actores que comenzaron a redefinir la seguridad desde sus particularidades, y finalmente, un proceso de asimilación

\footnotetext{
Pueden existir muchas diferencias sobre el concepto de seguridad. Pero en definitiva la seguridad se puede expresar como la garantía que provee el Estado. Después de la Segunda Guerra Mundial, Nacional Unidas trabajan para mantener la paz y seguridad internacionales, y bajo este precepto, la seguridad se constituye deber de las naciones para sus habitantes.
}

y aceptación de que existen fenómenos -no relacionados con la violencia en el sentido estricto-, pero que afectan la capacidad de supervivencia de las naciones. De igual manera, el artículo presenta la idea de necesidad de alinear la doctrina aérea con los objetivos en materia de seguridad y defensa de la Organización de Estados Americanos - OEA. Es pertinente iniciar el debate en este sentido, puesto que es la dirección más racional a fin de garantizar un correcto empleo del Poder Aéreo nacional en beneficio de la política de seguridad y defensa del Estado colombiano.

Con el fin de ofrecer una carta de ruta al propósito investigativo de este artículo, los autores proponen la pregunta problémica de: ¿Cómo se debe entender la seguridad en los inicios del siglo XXI, y cómo -desde un punto de vista concreto- adaptar los recursos del Poder Aéreo colombiano a los requerimientos y retos de la Seguridad Multidimensional?

Con relación al objetivo general y la pregunta problémica ya presentada, este escrito constará de tres objetivos específicos. El primero consiste en analizar la seguridad desde un ámbito clásico, o cuando las problemáticas frente a estas se definían únicamente a partir de las relaciones de poder entre los Estados-nación, y su capacidad de ejercer la guerra contra otros. En una segunda instancia, estudiar y enseñar las modificaciones que presentó la seguridad y las amenazas que su comprensión se deriva, y así poder generar claridad acerca del primer momento de transformación de este ambiente para los Estados. Tercero, presentar el concepto y debate más reciente sobre la seguridad, con el ánimo de interpretar porqué la noción contemporánea que tenemos de ella se encuentra estrechamente relacionada con conceptos como lo "humano" o "multidimensional".

A partir de estos tres ejes específicos planteados, se espera ofrecer al lector un proceso analítico comparativo y deductivo, que presente la idea de armonizar una dinámica de seguridad propia de la doctrina clásica de defensa del territorio y soberanía, a la nueva doctrina de seguridad, que admite dentro de sí misma nuevos fenómenos como pandemias, desastres naturales, las actividades que ponen en peligro el medio ambiente, los recursos naturales y los derechos humanos, entre otros. 
Por esto, es un documento investigativo que se genera del producto de la revisión bibliográfica de segundo orden, compuesta por artículos de investigación cientíica y académicos, pero también con base en documentos de primer orden que hacen referencia a adaptaciones institucionales que llevan a replantear la seguridad en nuestros días.

Finalmente, lo anterior condujo a la conclusión de la estrecha relación que hay entre el Poder Aéreo y la Seguridad Multidimensional, en el contexto de la seguridad y la defensa de la nación colombiana, en donde ambas doctrinas tienen afinidades comunes y su integración genera sinergia a los demás campos del poder militar y a los objetivos estatales en esa materia.

\section{Doctrinas de Poder Aéreo en Colombia y las amenazas tradicionales a la Seguridad Multidimensional}

La Seguridad Multidimensional reúne todos aquellos factores, conflictos o fenómenos que afectan la seguridad y desarrollo de las naciones. Desde las amenazas de una guerra entre estados, hasta el terrorismo, el problema mundial de las drogas, la corrupción, la minería ilegal y la pobreza extrema, entre muchos más, y esta última, como una de aquellas que puede detonar la violencia. Por ello, la OEA (2017a), en su preocupación por el desarrollo y la paz en el hemisferio sur, se ha convertido en referente para la cooperación entre los Estados, el fortalecimiento de las democracias y la lucha contra las amenazas a la paz. En este contexto, es necesario que el compromiso solidario de los Estados en materia de Seguridad Multidimensional se aborde con el enfoque dado por estos mismos en la OEA, y de esta forma enfrentar los temas de seguridad que afectan a cada país en el hemisferio sur. De otra parte, el Poder Aéreo desde el siglo pasado, ha mostrado a través del tiempo sus enormes capacidades en guerras y conflictos, en misiones humanitarias, en la reducción de las distancias entre los continentes, en el comercio y desarrollo económico de los países, entre muchos más. Pero en concreto, el Poder Aéreo se ha constituido en un punto de referencia para el crecimiento, seguridad y defensa de las naciones; como parte fundamental de la estructura de desarrollo y protección de los estados.

De tal forma, el Poder Aéreo como parte del poder militar de un Estado-nación, supera las capacidades terrestres y navales de los otros poderes, por cuanto su connotación tridimensional trasciende los límites de tierra y mar. En otras palabras, el Poder Aéreo "elimina la necesidad de confrontar los accidentes del terreno o el medio ambiente por su habilidad de volar sobre contingentes armados, flotas, y obstáculos geográficos, y atacar directamente a los centros claves del enemigo" (Meilinger, 1996).
En este mismo sentido, la doctrina en la Fuerza Aérea Colombiana ha evolucionado a través del tiempo, gracias a la experiencia, la tecnología y los medios que, en el tiempo, la han fortalecido. Es así como todos los conceptos teóricos de "empleo del Poder Aéreo y espacial en pro de la seguridad y defensa nacional y de los demás fines del Estado" (JEA, 2016, p. 17), están contenidos en el objetivo del Manual de Doctrina Básica Aérea (en adelante: MABDA). De esta forma, el interés del presente escrito es el de integrar ambas doctrinas que giran alrededor de la defensa y la seguridad.

De este modo es evidente la relación entre la Seguridad Multidimensional y el Poder Aéreo, muy estrecha por el contexto que define la primera y el compromiso del segundo en la totalidad de lo establecido en la doctrina de la OEA, así como las obligaciones y compromisos adquiridos por los Estados a partir del 2003, como se explica a continuación.

El primer uso del término de Seguridad Multidimensional se dio en la Asamblea General de Bridgetown en el 2002, cuando al criterio tradicional de seguridad, es decir, las amenazas de tipo militar fueron incorporadas nuevas problemáticas sociales, políticas, económicas, de seguridad humana (PNUD, 1994) y medio ambiente (Stein, 2017). Pero el concepto fue adoptado final y oficialmente por la OEA (2003), en la "Conferencia especial de Seguridad" efectuada en México, con la participación de todos embajadores de los Estados miembros, cuya conclusión se centró en su preocupación por la paz y la seguridad hemisférica, donde concluyeron que "la seguridad en el hemisferio es de alcance multidimensional" (OEA, 2003, p. 1) e incorporaron en sus discusiones, las nuevas amenazas (Delincuencia Organizada Transnacional - DOT), terrorismo y las vulnerabilidades sociales y ambientales) ${ }^{3}$, a las amenazas tradicionales (amenazas al territorio nacional y desafíos a la soberanía y la independencia)4.

Se puede deducir de lo anterior cómo el Poder Aéreo

\footnotetext{
${ }^{2}$ La seguridad humana tiene dos aspectos principales. En primer lugar, significa seguridad contra amenazas crónicas como el hambre, la enfermedad y la represión. En segundo lugar, significa protección contra alteraciones súbitas y dolorosas de la vida cotidiana, ya sea en el hogar, en el empleo o en la comunidad. Dichas amenazas pueden existir en todos los niveles de ingreso y desarrollo de un país.

${ }^{3}$ Nuevas amenazas: Delincuencia Organizada Transnacional (corrupción, lavado de activos, trata de personas, tráfico ilícito de armas y drogas y la conexión entre estas), terrorismo (acceso a las armas de destrucción masiva - ADM, ataques a la seguridad cibernética) y vulnerabilidades sociales y ambientales (pobreza extrema y exclusión social, desastres naturales de origen humano, VIH/Sida y otras enfermedades, deterioro del medio ambiente y accidentes de materiales peligrosos).

${ }^{4}$ Amenazas tradicionales: amenazas al territorio nacional y desafíos a la soberanía y la independencia.
} 
está inmerso en el compromiso para dar respuestas y soluciones a los problemas de la Seguridad Multidimensional, más aún, las teorías de los grandes pensadores del tema toman fuerza y permanecen aún más vigentes, puesto que refuerzan su papel fundamental en una doctrina propuesta por la OEA. Lo importante es hacer uso del Poder Aéreo en conjunción con la Seguridad Multidimensional, en un enfoque legítimo de los Estados, de primer orden y contundente en lo que se requiera. Lo anterior, daría validez al siguiente argumento:

El Poder Aéreo puede percibirse como una herramienta sofisticada y polivalente que puede ser usada contra cualquier tipo de enemigo, sea simétrico o asimétrico. La diferencia entre un éxito arrollador o un fracaso catastrófico en el uso de esta maravillosa herramienta reside en emplear la función adecuada para la necesidad concreta (...) (Palermo, 2006, párr. 43).

Sin embargo, el Poder Aéreo abarca mucho más que lo expresado por Palermo. Por ejemplo, como una herramienta empleada en toda su flexibilidad, la contribuye en situaciones de la seguridad humana en un rescate, una evacuación aeromédica, la extinción de incendios, el transporte de ayuda humanitaria y en un sinnúmero de misiones, convirtiéndolo en el mejor y más rápido instrumento para llegar a donde el ser humano lo necesite, más allá de las obligaciones de seguridad y defensa que también hacen parte de la Seguridad Multidimensional. Aquí se puede ver claramente un rol distinto al de defensa frente a las amenazas externas (tradicionales), el cual ya está circulando en las tareas de las FF. MM. de algunos países del mundo, especialmente de Colombia.

\section{El empleo del Poder Aéreo a las nuevas amenazas y sus características a partir de la globalización y aplicación de las TIC}

Ahora, esta doctrina de la Seguridad Multidimensional, abarca toda la problemática hemisférica de la violencia transnacional (sin que esto sea un límite por ser doctrina de la OEA, puesto que afecta al mundo entero), producto de desigualdades, deficiencias de los gobiernos en el manejo de la seguridad interna y desarrollo, como también la falta de oportunidades para los ciudadanos, la corrupción y las condiciones de pobreza extrema, entre otras razones, han originado en buena parte al incremento considerable de todas las formas de violencia; llegando inclusive a la estructuración de la Delincuencia Organizada Transnacional - DOT, y al terrorismo, fenómenos que se encuentran en circulación transfronteriza y a la que impactan directamente al desarrollo, la estabilidad y la libertad del hemisferio. Situaciones que hoy en día son preocupación global.
En este contexto, el uso del Poder Aéreo se hace necesario; no solo para conflictos entre los estados, sino, en un conflicto interno y asimétrico, que de acuerdo con el significado dado por el Mayor José Palermo en su documento Redefiniendo asimetrías en el empleo del Poder Aéreo, "se refiere a la manera y medios extremos de lucha que no corresponden a los objetivos y medios de combate del oponente, utilizando recursos diferentes a los del adversario" (2006, párr. 4). Sin embargo, el concepto de conflicto asimétrico debe ser diferenciado como lo menciona Palermo, del término guerra asimétrica, que por las características y las diferencias de poder e intensidad del conflicto como el caso colombiano; bien podría utilizarse el término, y que finalmente se define como aquel conflicto armado en gran escala donde la relación entre las partes implica una considerable diferencia respecto de los "poderes militares" en pugna.

Según Palermo, "los "enemigos asimétricos" son una realidad, están entre nosotros y la pregunta no es si los enfrentaremos, sino cuándo, cómo y dónde lo haremos" (2006, párr. 78). Frente a esto, el Poder Aéreo proporciona la flexibilidad, el alcance y la velocidad que militares y policías requieren para combatirlo, en el contexto de la legalidad en materia de derechosh, en beneficio de la Seguridad Multidimensional.

Ahora bien, frente a la problemática de seguridad, de la cual Colombia no es la excepción, la OEA recopiló las formas de afectación a la vida humana como objetivo central de la Seguridad Multidimensional, y ha trabajado desde mucho tiempo atrás en la integración permanente de los Estados en la conformación de alianzas y cooperación contra las vulnerabilidades y preocupaciones a fin de proteger al ser humano, como lo son las medidas del fomento de la confianza, acordadas en la declaración de Santiago (OEA, 1995), para "afianzar la paz y la seguridad en el hemisferio" (párr. 1), así como los Libros Blancos, incluidos en las citadas medidas de confianza.

De lo anterior, se puede deducir cómo la problemática de Seguridad Multidimensional no solo implica a militares y policías; ellos tan solo son una parte de un acuerdo suscrito por los presidentes de los Estados, quienes pactan tomar medidas que enfrenten la problemática de seguridad y defensa de sus países. De tal forma que el Poder Aéreo es parte fundamental en ese orden, como fragmento de la sinergia que el Poder Militar reúne para cumplir con las necesidades constitucionales para la preservación de la seguridad y la defensa del país. Sin embargo, la sinergia desde el punto de vista del Poder Aéreo, según Frederick Baier en su artículo Cincuenta preguntas que todo aviador puede contestar, se describe como: 
La idea de que la combinación de diferentes capacidades crea efectos más potentes que cuando se usan de forma individual. Sinergia es crecimiento exponencial de efecto, no crecimiento lineal. Cuando los aviadores miramos el potencial del poderío aeroespacial para crear efectos, rápidamente nos damos cuenta que los efectos secundarios, terciarios y sucesivos (a menudo descritos como efectos en "cascada") resultan de la aplicación sinergista de las diferentes capacidades del poderío aeroespacial. Cada una de nuestras capacidades fundamentales resulta de la naturaleza sinergista del poderío aeroespacial (2005, párr. 30).

La anterior afirmación, demuestra aún más, la importancia de primer orden que el Poder Aéreo tiene a la hora de potencializarse en la conjunción, siendo un elemento de enormes capacidades.

Es así como la Seguridad Multidimensional y el Poder Aéreo, guardan una estrecha relación en lo que se refiere a la ser humano, su protección, por el solo derecho que tiene a la seguridad. En este argumento, la Declaración de la Seguridad de las Américas de la OEA en el 2003 (OEA, Conferencia Especial sobre Seguridad, 2003), refiere lo siguiente:

El fundamento y razón de ser de la seguridad es la protección de la persona humana. (...) Las condiciones de la seguridad humana mejoran mediante el pleno respeto de la dignidad, los derechos humanos y las libertades fundamentales de las personas, así como mediante la promoción del desarrollo económico y social, la inclusión social, la educación y la lucha contra la pobreza, las enfermedades y el hambre, (...) el concepto y los enfoques tradicionales deben ampliarse para abarcar amenazas nuevas y no tradicionales, que incluyen aspectos políticos, económicos, sociales, de salud y ambientales. (p. 3)

En este enfoque dado a la seguridad en el hemisferio de carácter multidimensional y aclarando un poco acerca del fundamento de la protección de los seres humanos, manifestado en las declaraciones de la OEA en ciudad de México en la Conferencia Especial sobre Seguridad, 2003 y Nuevo León en la Declaración de Nuevo León, (2004), aparecen dos términos asociados y parte de la Seguridad Multidimensional: la seguridad humana ${ }^{5}$ la cual se trató al

\footnotetext{
${ }^{5}$ Seguridad humana: de acuerdo con el concepto de la Secretaría de Seguridad Multidimensional de la OEA del 2011, "tiene dos aspectos principales. En primer lugar, significa seguridad contra las amenazas crónicas como el hambre, la enfermedad y la represión. En segundo lugar, significa protección contra alteraciones súbitas y dolorosas de la
}

comienzo de este escrito y la seguridad nacional ${ }^{6}$, que son necesarios conocer para mejorar los conocimientos en el tema, en la legitimización del uso del poder militar.

La seguridad humana se enfoca en el individuo como "preocupación universal" (PNUD, 1994, p. 22),"por la vida y la dignidad humana"(PNUD, 1994, p. 25), que adquiere el valor hemisférico multidimensional; alineado con sus libertades respecto al miedo y sus necesidades. Un ejemplo clásico de las preocupaciones de la seguridad humana viene de Albert Einstein después de la detonación de la bomba nuclear al expresar si la "humanidad ha de sobrevivir" (PNUD, 1994, p. 25), pues se había descubierto la mejor forma de acabar con los seres humanos. Y la seguridad ciudadana como parte de la seguridad humana, va dirigida a la seguridad de las personas dentro del Estado (OEA, Comisión Interamericana de Derechos Humanos, 2017), frente a la defensa del territorio y la soberanía. Como se citó anteriormente, los dos conceptos están ligados a la Seguridad Multidimensional y fueron motivo de definición por parte de la OEA en la estructuración general de la preocupación por la paz y seguridad en el hemisferio, mencionado anteriormente.

\section{La armonía de empleo del Poder Aéreo frente a los escenarios de preocupaciones y nuevos desafíos a la seguridad}

Por otra parte, y a fin de entender "las amenazas, preocupaciones y otros desafíos" (OEA, Conferencia Especial sobre Seguridad, 2003) de la Seguridad Multidimensional, es necesario retomar lo explicado anteriormente, en el sentido de que las amenazas se clasifican en dos: las amenazas tradicionales y las nuevas amenazas. Las primeras (amenazas tradicionales) concebidas desde tiempo atrás, son aquellas agresiones contra los países y sus ciudadanos, provenientes de "fuerzas externas" o amenazas propias de la defensa de las naciones (Blackwell, 2017).

Hay una estrecha relación entre defensa y seguridad (amenazas tradicionales y nuevas amenazas), si se quiere analizar desde una óptica más sencilla de entender. Guzmán define ambos conceptos de esta forma:

Seguridad (...) como todas aquellas actividades necesarias para proteger los intereses del país cuando este se ve enfrentado a riesgos y amenazas o interferencias que afecten su desarrollo

vida cotidiana, ya sea en el hogar, en el empleo o en la comunidad. La seguridad humana siempre ha tenido dos componentes fundamentales: libertad respecto del miedo y libertad respecto de la necesidad".

6 Seguridad nacional: de acuerdo con el concepto de la Secretaría de Seguridad Multidimensional de la OEA del 2011, debe entenderse como la seguridad del Estado, es decir, la seguridad de todas las personas que lo conforman. 
social y económico. Es así como la defensa contribuye a la seguridad del país tanto por medio de su preparación para el empleo legítimo de la fuerza, su contribución a la disuasión y la participación de sus medios en misiones de cooperación internacional (Guzmán, 2013, p. 76).

De esta manera, el Poder Aéreo se convierte en un "elemento clave para lograr la parálisis estratégica y la subsecuente victoria militar" (Valente, 2017, párr. 13), sobre un enemigo, en función de seguridad y defensa y como herramienta de desarrollo nacional, denotando así que las capacidades aéreas de una nación deben estar puestas al servicio de todas esas necesidades de protección que el ser humano requiere; en el tiempo, lugar y modo requerido y de forma oportuna e inmediata.

No obstante, en temas de protección en términos de amenazas tradicionales y nuevas amenazas de la Seguridad Multidimensional, es necesario incluir conceptos de la doctrina aérea como la superioridad aérea ${ }^{7}$, indispensable para que el Poder Aéreo pueda desenvolverse con"libertad de acción" (Baier, 2005), seguridad y tranquilidad en su propio territorio, a fin de proveer las condiciones de seguridad y defensa que la Seguridad Multidimensional requiere, porque quien controla el espacio aéreo generalmente controla el terreno (Meilinger, 1996). En términos más concretos, una relación en que la superioridad aérea es un objetivo del Poder Aéreo para actuar en garantía a la Seguridad Multidimensional.

Es así como la superioridad aérea soluciona problemas de Seguridad Multidimensional, al ser un elemento disuasivo frente a las amenazas tradicionales y nuevas amenazas, un factor de desequilibrio sicológico a las acciones que otro país o la DOT y el terrorismo, puedan llegar a cometer contra el propio Estado, coadyuvando entre muchas y quizá la más importante: la parálisis estratégica del adversario, entendiendo esto "como la imposibilidad física del enemigo para oponerse a nuestra voluntad. (...) Negando al comando enemigo la capacidad de responder racionalmente y de esa manera hacerle incapa" (Valente, 2017), de come-

\footnotetext{
${ }^{7}$ Superioridad aérea es un estándar relativo de libertad de acción que describe la capacidad de conducir operaciones aéreas contra un adversario sin que las fuerzas del adversario opongan obstáculos insuperables a nuestras acciones. La superioridad aérea es una escala deslizante basada en medidas objetivas y factores subjetivos; su presencia o ausencia está determinada por el criterio y experiencia del comandante apropiado, a menudo suplementada por recomendaciones de sus oficiales o subordinados. La historia nos indica que en última instancia la superioridad aérea proporciona mucho más que la simple libertad de operación a las fuerzas aeroespaciales. Proporciona a la totalidad de la fuerza conjunta la condición de estar libres de ataques, la libertad de maniobra y la libertad de atacar (Baier, 2005).
}

ter acciones en contra de los propios interese en materia de seguridad y defensa.

Con base en lo anterior, y frente a la evolución de amenazas emergentes (Blackwell, 2017) , así como el incremento de la violencia en el hemisferio americano y dicho anteriormente, se dio origen a las llamadas nuevas amenazas (DOT en todas sus formas y el terrorismo). La inseguridad debido a la DOT es producto de afectaciones e implicaciones (Costa, 2017) directas en lo político, al no lograr obtener la confianza ciudadana con resultados por parte de los gobiernos, en lo económico por los costos de la violencia y su impacto en las economías y proporción del PIB de los países, presentados por el Banco Interamericano de Desarrollo en materia de seguridad ciudadana (Marmolejo, 2014), en derechos humanos por la falta de las garantías estatales para el respeto de estos y en salud pública por los riesgos a la vida e integridad de las personas.

Ahora bien, el terrorismo puede llegar a ser una forma mucho más violenta que la DOT, se puede ver desde el acceso a las Armas de Destrucción Masiva (ADM), los ataques a la seguridad cibernética y los ataques al ser humano: muerte y terror. Es así como los conceptos anteriores se consideran elementos constitutivos de impacto transfronterizo, y cuyos ataques están dirigidos sin distinción contra la población, constituyéndose en riesgos transnacionales de preocupaciones para los Estados del mundo. Este llamado terrorismo es de carácter criminal y "despliegue asimétrico" (Tilly, 2004, p. 5), de amenazas y violencia fuera de control político con propósitos ilegales, dirigidos a buscar terror (Tilly, 2004) en la población; sin fronteras (campo de batalla propio acorde a sus capacidades de alcance) y de difícil supresión de los Estados.

Este terrorismo es combatido en todo el mundo. Por tal razón, el escenario actual de la lucha global contra esta amenaza se constituye en uno de los principales objetivos de disputa por la seguridad en los países afectados por las bombas, decapitaciones, violaciones, secuestros, tráfico humano y muchos otros actos de muerte y terror, que son combatidos en todos los campos del poder, pero principalmente del Poder Aéreo, a través de la superioridad aérea, que brinda mayor efectividad y protección a las tropas que combaten por tierra la misma amenaza, sin demeritar los grandes esfuerzos en tierra y superficie en la conjuntes operacional que ha sido efectiva en Colombia en los últimos años, y que tiene a militares y policías nacionales en los primeros órdenes de confianza por su sacrificio.

De las situaciones anteriores: DOT y terrorismo como parte de las nuevas amenazas y el tema de preocupaciones y desafíos que se tratará más adelante, Víctor Guzmán 
(2013), en su artículo cita a David Pion-Berlin en Militares y democracia en el nuevo siglo, acerca del rol de los militares (incluido el Poder Aéreo) y en general de las Fuerzas Armadas (en adelante: FF. AA.), el cual será ampliado con mayores responsabilidades frente a temas internos como los mencionados anteriormente y que van más allá de su rol tradicional, de la siguiente forma:

Muchas situaciones extremas, donde los gobiernos suelen verse en apuros, terminan con la solicitud del poder político, es decir de la civilidad, para que las FF. AA. intervengan en asuntos específicos, sea para controlar situaciones de violencia extrema en sectores de una ciudad o para enfrentarse a las bandas de narcotraficantes, o bien para atender desastres naturales que sobrepasan las capacidades civiles, una tendencia generalizada que fue analizada en términos positivos en la reunión de los ministros de Defensa en noviembre de 2010 (Guzmán, 2013, p. 180).

Es claro que en el futuro esta situación, especialmente en el hemisferio sur, se estará viendo con la mayor normalidad, como actualmente se observa en Colombia; no solo en temas de catástrofes y temas medioambientales de las preocupaciones y desafíos multidimensionales, sino en las operaciones contra estas organizaciones, como parte de una doctrina que ha venido evolucionando, como es el caso del combate contra el narcotráfico, la minería criminal, estructuras criminales, entre muchas, citadas al inicio de este escrito.

A propósito del narcotráfico como la principal fuente de recursos para la DOT y el terrorismo, Guzmán argumenta:

Involucrar a las FF. AA. en la lucha contra la droga requiere de cambios doctrinales y de misión militar, añadiendo la dificultad adicional de que el éxito final dependerá de la calidad de la democracia y solidez institucional de cada país, toda vez que uno de los objetivos de las bandas de narcotraficantes es ir creando "espacios vacíos" donde imponer su autoridad para crear parcelas de su propia legitimidad (2013, p. 181).

Ante este panorama que genera mayores responsabilidades al rol de los militares, incluyendo al Poder Aéreo, "es necesario un rediseño del sistema que sea capaz de manejar las redes globales y las complejidades multidimensionales que emergen en un constante sinfín" (Guzmán, 2013, p. 194). Lo anterior implica: acoger la Seguridad Multidimensional como doctrina; no solo en terminología, sino en la arquitectura de la seguridad constituida por la OEA para enfrentar todas las amenazas a la seguridad, para así generar una respuesta hemisférica, regional y con aporte global a estas amenazas transnacionales que afectan hoy en día al planeta entero. Es también cumplir con las obligaciones pactadas, acudir a las reuniones de ministros de Defensa a trabajar en beneficio de la seguridad del hemisferio con resultados globales y adoptar las medidas de confianza que garanticen, entre otras, la cooperación para el desarrollo, el combate a las amenazas transnacionales y fortalecer la mencionada seguridad.

Por otro lado, están las vulnerabilidades sociales y ambientales (pobreza extrema, exclusión social, desastres naturales y de origen humano, el Sida y otras enfermedades, el deterioro del medio ambiente y accidentes de materiales peligrosos), que se constituyen en las preocupaciones y desafíos multidimensionales. Estas vulnerabilidades de los estados afectan el desarrollo nacional (bienestar), el sentido humanitario (las emergencias) y seguridad (amenazas y riesgos). La pobreza extrema y exclusión social, los desastres naturales y de origen humano, el VIH/Sida y otras enfermedades y el deterioro del medio ambiente, son entre otros, desafíos en materia de seguridad, puesto que la efectividad de la respuesta estatal o no, genera la focalización de escenarios de protesta, violencia y profundas divisiones sociales.

Es en este escenario multidimensional, el Poder Aéreo juega un papel importante y que va más allá de su empleo como arma. Tal como lo argumenta "John Warden, el Poder Aéreo transporta información estratégica: alguna es negativa (como las bombas), y otra es positiva (como los alimentos)" (Meilinger, 1996, párr. 19). Desde esta perspectiva, la Seguridad Multidimensional pasa a ser beneficiada por las capacidades del Poder Aéreo.

Independientemente de un posacuerdo, lo cierto es que en el caso de Colombia, la necesidad de enfrentar el terrorismo y mantener la democracia, los buenos niveles de economía y un aceptable desarrollo social, requiere que el empleo de los militares multiplique la labor de la Policía Nacional, mejorando la seguridad, la confianza y fortaleciendo las políticas de seguridad (Blackwell, 2017).

Finalmente, en este sentido, en los países del hemisferio sur, puede observarse una lista extensa de preocupaciones y desafíos de la Seguridad Multidimensional, donde el Estado tiene la principal obligación en materia de protección y seguridad, en la constitución de políticas de Estado, gobierno y públicas, a fin de garantizar las condiciones de vida digna de sus ciudadanos. De tal forma, el Poder Aéreo como parte del poder militar, debe actuar de manera efectiva en la solución de esta problemática social que afecta la seguridad, mediante la conducción de operaciones a todo 
tiempo como: el transporte de material y personal en situaciones de catástrofes de origen natural o las producidas por el mismo hombre: rescates, transporte aeromédico, extinción de incendios, cooperación en trabajos de infraestructura, etc.

\section{Conclusiones}

Los problemas de violencia en el hemisferio han sido agrupados y ordenados dentro de un nuevo orden multidimensional, que busca analizar la situación de violencia de los países Latinoamericanos como Colombia, para dar solución en prevención y superación de las preocupaciones frente al alto riesgo a la vida, enfocando el esfuerzo al ser humano, más allá de las sociedades y los estados en los que habite.

La respuesta de los estados a la "cultura de la trasgresión" (poder instalado y menosprecio del pueblo) (Sorj y Martuccelli, 2017, p. 126), debe enfocarse a la planificación de políticas de seguridad humana, fortalecimiento de las relaciones internacionales, mediante acuerdos en materia de seguridad que permitan alcanzar el desarrollo socioeconómico y reduzca las amenazas y riesgos a la vida del individuo.

Las respuestas institucionales y estatales frente a las amenazas, partiendo del desarrollo y crecimiento de la problemática criminal como sucede en Colombia, ha llevado a los Estados a una reestructuración en políticas de seguridad como el "Consenso de Santo Domingo sobre Seguridad Pública" (OEA, segunda reunión de ministros en materia de Seguridad Pública de las Américas MISPA II, 2009), donde los ministros de Defensa o Seguridad concuerdan en la implementación de un programa en materia de seguridad en cada Estado, y avanzar en aspectos de información y mutua cooperación en temas de Seguridad Multidimensional.

En este escenario hemisférico, se da la integración y participación directa de las Fuerzas Militares en operaciones de orden interno en los Estados como en Colombia, en donde el espectro operacional ha alcanzado mayores niveles en beneficio de la población, víctima de todas las amenazas multidimensionales, inclusive el terrorismo, que en más de 50 años ha azotado al pueblo colombiano, con lamentables pérdidas de vidas no solo de civiles, sino también de militares y policías en su función de seguridad (Blackwell, 2017).

Un aspecto importante es el de la participación del Poder Aéreo (incluido en el poder militar) en sacar adelante el programa de los 17 "Objetivos de Desarrollo Sostenible" (Naciones Unidas, Objetivos de Desarrollo Sostenible,
2015), considerados por la misma Organización de las Naciones Unidas como un "conjunto de objetivos globales para erradicar la pobreza, proteger el planeta y asegurar la prosperidad para todos como parte de una nueva agenda de desarrollo sostenible" (ONU, 2015, p. 1).

De lo anterior, el rol de militares y policías también es otro; no solamente lo acostumbrado en términos de conflicto externo o interno (amenazas tradicionales y nuevas amenazas), sino, precisamente las preoculaciones y desafíos de la Seguridad Multidimensional, que implica compromiso en sacar adelante proyectos de desarrollo social y la conducción de operaciones aéreas por la vida y dignidad humana.

El Poder Aéreo y la Seguridad Multidimensional tienen una relación estrecha que conlleva a un planteamiento del mapa de la seguridad y la defensa de una nación como Colombia; involucrando dos doctrinas que potencializan el actuar de un Estado en la solución de conflictos externos, internos y la problemática social interna, en términos más sencillos: la adaptación del Poder Aéreo colombiano; doctrina, capacidades y medios, a la Seguridad Multidimensional establecida por la OEA desde el 2002.

Todo lo anterior se convierte en una forma de innovar hacia la transformación unificada de conceptos que ya existen. Solo es mezclarlos y sacar de ellos el mejor provecho. A propósito de la innovación de los militares, el expresidente norteamericano George Bush plantea:

La formación de una institución militar del siglo XXI demandará más que nuevas armas. También demandará un espíritu de innovación renovado de nuestros cuerpos de oficiales. No podemos transformar nuestros servicios militares usando armas antiguas y planes obsoletos. Ni podemos hacerlo con un modo de pensar burocrático anticuado que frustra la creatividad y el espíritu emprendedor que necesitarán los servicios militares del siglo 21 (McNerney, 2005).

El Poder Aéreo y en general, el poder militar, debe ser cada día más innovador, conforme a las necesidades de un Estado, pero con la convicción de que el gobierno sea su padrino en la transformación y cambios que este debe tener, acorde a la tecnología, medios y economía necesaria para mantenerlo y constituirlo como uno de los mayores bienes de patrimonio, que permite soluciones a la Seguridad Multidimensional. 


\section{Referencias}

Baier, F. (2005). Cincuenta preguntas que todo Aviador puede Contestar. Air \& Space Power Journal. Recuperado de http://www. airpower.maxwell.af.mil/apjinternational/apj-s/2005/3tri05/ baier.html

Blackwell, A. (2017). Seguridad Multidimensional: "enfrentando nuevas amenazas". Recuperado de https://adamblackwell. wordpress.com/2014/07/23/seguridad-multidimensionalenfrentando-nuevas-amenazas/

Costa, G. (2017). Seguridad ciudadana y delincuencia organizada transnacional en las Américas: situación y desafíos en el ambito interamericano. Revista Internacional de Derechos Humanos, 9(16), 133. Recuperado de http://www.conectas.org/es/ acciones/es-revista-sur/edicion/16/544-seguranca-publicae-crime-organizado-transnacional-nas-americas-situacao-edesafios-no-ambito-interamericano

Guzmán, V. M. (2013). Rol y funciones de las Fuerzas Armadas en el siglo XXI: un enfoque desde las nuevas estrategias de seguridad. En A. N. Anepe (Editor), Revista Política y Estrategia, 232. Santiago de Chile: Julio E. Soto Silva e Iván Rojas Coromer.

JEA. (2016). Manual de doctrina básica aérea y espacial, cuarta edición. Bogotá: Departamento Fuerza Aérea ESDEGUE.

Marmolejo, M. D. (2014). 5 Aspectos claves del trabajo del BID en Seguridad Ciudadana. Washington: BID.

McNerney, M. (2005). Innovación militar en tiempos de conflicto. ¿Es demasiado riesgoso? Air \& Space Power Journal. Recuperado de http://www.airpower.maxwell.af.mil/apjinternational/apj-s/2005/2tri05/mcnerney.html

Meilinger, C. P. (1996). Diez propuestas en relación con el Poder Aéreo. Air \& Space Power Journal. Recuperado de http://www.au.af.mil/au/afri/aspj/apjinternational/apjs/1996/4trimes96/meilinger.html

OEA. (1995). Decaración de Santiago sobre medidas de fomento de la confianza y de la seguridad. Consejo Permanente de la Organización de los Estados Americanos. Recuperado de http://www.oas.org/csh/spanish/mfcdeclsant.asp

OEA. (2003). Conferencia especial sobre seguridad. Recuperado de http://www.oas.org/36AG/espanol/doc_referencia/DeclaracionMexico_Seguridad.pdf
OEA. (2004). Declaración de Nuevo León. Recuperado de https:// www.oas.org/36ag/espanol/doc_referencia/CumbreAmericasMexico_DeclaracionLeon.pdf

OEA. (2009). Segunda reunión de ministros en materia de Seguridad Pública de las Américas MISPA II. Recuperado de http://www. oas.org/dsp/espanol/cpo_mispa2.asp

OEAa. (2017). Organización de los Estados Americanos. Carta Democrática Interamericana. Recuperado de http://www.oas. org/OASpage/esp/Documentos/Carta_Democratica.htm

OEAb. (2017). Informe sobre seguridad ciudadana y derechos humanos. Recuperado de https://www.oas.org/es/cidh/docs/ pdfs/SEGURIDAD\%20CIUDADANA\%202009\%20ESP.pdf

ONU. (2015). Objetivos de desarrollo sostenible. Recuperado de https://www.unric.org/html/english/library/backgrounders/ sdgs_spanish.pdf

Palermo, J. (2006). Redefiniendo Asimetrías en el Empleo del Poder Aéreo. Obtenido de Air \& Space Power Journal: http://www. au.af.mil/au/afri/aspj/apjinternational/apj-s/2006/2tri06/pa lermo.html

PNUD. (1994). Informe sobre desarrollo humano 1994. México: Fondo de Cultura Económica, S. A. de C. V. Recuperado de http://hdr.undp.org/sites/default/files/hdr_1994_es_completo_nostats.pdf

Sorj, B., y Martuccelli, D. (2017). III. Problemas y promesas: economía informal, crimen y corrupción, normas y derechos. En Centro Edelstein de Pesquisas Sociais (Ed.), El desafio latino-americano: cohesión social y democracia (pp. 125-167). Recuperado de http://books.scielo.org/id/4fdcs/pdf/sorj-978857982 0793-04.pdf

Stein, A. (2017). El concepto de Seguridad. Recuperado de https:// www.peaceportal.org/documents/13 0226273/130473587/ $\mathrm{El}+$ Concepto+de+Seguridad+Multidimensional/61 ea987948a6-464e-985c-8b75354eda21

Tilly, C. (2004). Terror, terrorism, terrorists. Sociological Theory, Theories of Terrorism: A Symposium, 22(1), 5-13.

Valente, D. O. (2017). La campaña aérea como teoría del Poder Aéreo y su aplicabilidad en Latinoamérica. Air Power Journal Internacional. Recuperado de http://www.airpower.maxwell. af.mil/apjinternational/apj-s/1997/3trimes97/valealas.htm 